\title{
Clinical Analysis of Nonfungal Nail Disease Among Children and Adolescents- a Descriptive Cross-Sectional Study
}

\author{
Sushmita Pradhan ${ }^{1}$, Yaling Dai ${ }^{2}$, Lin Xiong ${ }^{1,}$ * \\ ${ }^{1}$ Department of Dermatovenereology, West China Hospital, Sichuan University, Chengdu, China \\ ${ }^{2}$ Department of Laboratory Medicine, West China Hospital, Sichuan University, Chengdu, China
}

Email address:

sush_pradhan@hotmail.com (S. Pradhan), 839894198@qq.com (Yaling Dai), xionglin2k@163.com (Lin Xiong)

*Corresponding author

\section{To cite this article:}

Sushmita Pradhan, Yaling Dai, Lin Xiong. Clinical Analysis of Nonfungal Nail Disease Among Children and Adolescents- a Descriptive Cross-Sectional Study. Science Journal of Clinical Medicine. Vol. 6, No. 4, 2017, pp. 46-52. doi: 10.11648/j.sjcm.20170604.11

Received: May 29, 2017; Accepted: June 12,2017; Published: July 26, 2017

\begin{abstract}
Background: Nonfungal nail disease in children and adolescents is a limited part of general practice. There is a wide spectrum of viral, traumatic, infectious, inflammatory and congenital conditions that may affect the nail condition in children and adolescents. Therefore assessing nonfungal nail disease and its factors will help the practitioner to recognize and treat the nail disease and determine the necessity to refer a dermatologist when indicated. This study is aimed to assess the magnitude of nonfungal nail disease, to identify the clinical characteristics and factors associated with nonfungal nail disease in children and adolescents from 1 to 18 years old. Methods: The data were collected from all cases with nonfungal nail diseases with the use of descriptive cross-sectional study, under the age of 19 years old in West China Hospital, Sichuan University. Interview, nail sample for direct microscopy and culture along with clinical pictures were taken. Finally collected data was analyzed using the Software Package for Social Science (SPSS) version 21. Results: A total of 187 patients fulfilled the inclusion criteria for the study. The mean age of the cases was 5.5 years $(\mathrm{SD} \pm 3.8)$, female $96(51.3 \%)$ and male 91 $(48.90 \%)$. The majority of $113(60.40 \%)$ were at the age category $<5$ years of age. A large number of patients, $175(93.6 \%)$ were residing in the urban area. Around $77(41.20 \%), 44(23.50 \%), 37(19.78 \%)$ cases were identified to have a history of the common cold disease, hand-foot-mouth disease, and skin diseases, respectively. Among all cases, the magnitude of onychomadesis was 50 (26.70\%). On binary logistic regression age (P value 0.001$)$, hand-foot-mouth disease (P value 0.0001$)$ and skin disorder ( $\mathrm{P}$ value 0.020$)$ were significantly associated with onychomadesis. Finally on multiple logistic regression only age and hand-foot-mouth disease were significantly associated at, $(\mathrm{P}$ value 0.006$)$ and $(\mathrm{P}$ value 0.0001$)$ respectively. Conclusion: This study determines that the occurrence of nonfungal nail disease decreases with the increase in the age of children. Onychomadesis was the most common nonfungal nail disease found in children and it affected both the hands. The age and hand-foot-mouth disease were statistically significant to onychomadesis. However, more powerful and precise studies are needed in future.
\end{abstract}

Keywords: Nonfungal, Nail Disease, Children, Adolescents, Clinical Analysis, Descriptive Cross-Sectional Study, Onychomadesis

\section{Introduction}

Nail disorders mark a significant portion in the dermatologic conditions and bring up crucial challenges to the clinicians with distinct characteristics and presentations of nail diseases that require different treatment methods in children [1]. Nail disease in children can be due to congenital and hereditary malformations, infections, tumors, inflammatory processes and systemic diseases [2]. As of today, there is only a few research that has tried to assess the magnitude, factors and clinical features of nonfungal nail disease in children and adolescents in the world. However, some studies indicate that prevalence of nail conditions in the pediatric population was $3 \%$ to $11 \%[3$, 4]. Another study done in China demonstrated that among 98 
nail disease patients, 40 (41\%) patients were found to have nonfungal nail diseases and clinically found that nonfungal nail diseases were more common in children [5]. A clinical study analyzing 23 children of nonfungal nail diseases according to age, sex and nail disorder showed 1 child with twenty nail dystrophy, 3 with onychocryptosis, 1 with onychomadesis, 3 with Beau's line, 1 with median nail dystrophy, 5 with linear nails, 4 with pitted nails and 5 with longitudinal melanonychia [6]. Similarly, another recent study clinically analyzed 170 children with nonfungal nail dystrophy. Among them, 35 children had proper nutrition, 123 had normal nutrition and 12 had poor nutrition. 78 children lived in urban areas whereas 92 lived in rural areas. 93 children disliked vegetables, 46 disliked meat and 31 were found to have a balanced diet. 116 children were found with nail biting habits and 54 did not have nail biting habits. 152 children were not affected with any other diseases whereas 18 had other diseases, among which 12 were reported with eczema, 4 with lichen planus and 2 with atopic dermatitis. In 107 children, fingernails were affected, 46 with toenails and in 17 both finger and toenails were affected. Among them, the most common nail disease was 31 children with nail pitting, 28 with brittle nails oronychorrhexis and 27 patients with leukonychia. In serum trace element examination, 56 children showed a decrease in calcium, 36 with a decrease in zinc and18 with a decrease in iron [7].

Onychomadesis, onycholysis, nail pitting, nail discoloration, trachyonychia, lichen planus, nail thickening, etc are the important nonfungal nail diseases associated with different diseases like eczema, alopecia areata, hand-foot-mouth diseases, lichen planus, psoriasis etc. Therefore clinical analysis of nonfungal nail diseases among children and adolescents tend to highlight the distribution of nonfungal nail disease in different categories.

\section{Aim of the Study}

This study is aimed to assess the magnitude of nonfungal nail disease, to identify the clinical characteristics and factors associated with nonfungal nail disease in children and adolescents from 1 to 18 years old.

\section{Method and Materials}

\subsection{Study Setting}

Sichuan is a province in southwest China occupying most of Sichuan basin between the Himalayas on the west, the Daba in the north, and the Yungui Plateau to the east. Chengdu is a sub-provincial city serving as the capital city of Sichuan province. Chengdu has almost 191 hospitals. Among them, West China Hospital, Sichuan University in Chengdu was chosen as the study setting for the study. The purpose of selecting this hospital was its popularity and high patient flow.

\subsection{Study Design}

Using cross-sectional study design all eligible case's guardians were interviewed with a structured questionnaire. The sample was taken from the affected part of the nail for direct microscopy and culture. Finally, clinical picture was taken to identify and classify the types of nail disease.

\subsection{Study Population and Sampling Method}

The study population in this study was children and adolescents who visited West China Hospital during the period from June 2015 to January 2017, under 19 years old, who complained of nail diseases and confirmed as nonfungal nail infection by direct microscopy and culture.

\subsection{Data Collection Methods}

The questionnaires used to collect data contain socio-demographic characteristics of age, gender, place of residence, education; history of illness like a hand-foot-mouth disease, common cold, skin diseases and others consisting of the habit of nail biting, serum trace element level, the location of nail disease and clinical characteristics of nonfungal nail disease. Finally, nail clippings and scrapings technique was used to collect a sample for direct microscopy and culture.

Direct microscopy: The nail clippings included debris scraped from under the distal end of an infected nail and scrapings of the surface of the nail plate. The material was placed on a glass slide with one or two drops of $10 \%$ potassium hydroxide (KOH) (SIGMA company) covered with a coverslip within 2 hours observed by direct microscopy under $10 \mathrm{X}$ and $40 \mathrm{X}$ magnifications. $\mathrm{KOH}$ helps to dissolve the cellular and proteinaceous nonfungal material.

Fungal culture: The specimen was inoculated into a medium such as Sabouraud's dextrose agar containing cycloheximide and chloramphenicol (ZHWY-2102C Company) and incubated at $26^{\circ} \mathrm{C}$ for 28 days. The negative result from both the direct microscopy and the culture was included in the study. The examinations were carried at Department of Laboratory Medicine, West China Hospital, Sichuan University, Chengdu, China.

\subsection{Ethical Considerations}

Ethical clearance was sought from the Department of Dermatovenereology, West China Hospital, Sichuan University. Then, the information was given to study participant and caretakers regarding the aim of the study and based on that information verbal consent was taken from all participants.

\subsection{Statistical Analysis}

A total of 201 data were collected within the study period of June 2015 to January 2017. Finally, 187 cases were included in the analysis. Data were examined for completeness before entering into the software. Then, data entry analysis was performed using SPSS 21.0 statistics software (SPSS Inc.; Chicago, IL, USA). The descriptive statistics like frequency and mean was used to describe the variable. The binary logistic regression was used to analyze 
the association of independent variables. Then significant variables on binary logistic were reanalyzed on multiple logistic regressions. Finally to identify significant independent variable with P-value of 0.05 was done.

\section{Result}

\subsection{Socio-demographic Characteristics}

Within a period of one and a half year of data collection [June 2015- January 2017], a total number of 201 cases were identified. Among them, $14(6.90 \%)$ cases were excluded because of positive findings for fungal infection under direct microscopy and/or culture. The socio-demographic characteristics show that among 187 cases, 96 (51.30\%) were female and $91(48.70 \%)$ were male. The mean age of cases was 5.5 years $(\mathrm{SD} \pm 3.8)$. The results revealed that in majority 113 $(64.40 \%)$ cases were $<5$ years of age, $175(96.30 \%)$ reside in an urban area and $65(34.80 \%)$ with primary school education.

Table 1. Socio-demographic characteristics of the study participants with nonfungal nail disease. West China Hospital, Sichuan University $(N=187), 2017$. (Table 1)

\begin{tabular}{llll}
\hline Variable & Category & Freq(N) & Percentage(\%) \\
\hline \multirow{3}{*}{ Age } & 1-5 years & 113 & 60.40 \\
& 6-12 years & 59 & 31.60 \\
Gender & 13-18 years & 15 & 8.00 \\
& Male & 91 & 48.90 \\
Residence & Female & 96 & 51.30 \\
& Urban & 175 & 93.60 \\
\multirow{3}{*}{ School/Education } & Rural & 12 & 6.40 \\
& No education & 58 & 31.00 \\
& Kindergarten & 52 & 27.80 \\
& Primary school & 65 & 34.80 \\
& Secondary & 12 & 6.40 \\
\hline
\end{tabular}

\subsection{History of Illness}

The past and present history of illness were assessed and among 187 cases 77 (41.20\%), 44 (23.50\%), 37 (19.78\%) cases were identified with the history of common cold, history of hand-foot-mouth disease and history of skin disease respectively. (Table 2)

Table 2. History of illness of the study participants with nonfungal nail disease. West China Hospital, Sichuan University ( N=187), 2017.

\begin{tabular}{llll}
\hline Variable & Category & Frequency(N) & Percentage(\%) \\
\hline Common cold & & 77 & 41.20 \\
HFM disease & & 44 & 23.50 \\
& Eczema & 25 & 13.40 \\
\multirow{2}{*}{ Skin disease } & Urticaria & 2 & 1.10 \\
& Psoriasis & 2 & 1.10 \\
& Others & 8 & 4.20 \\
\hline
\end{tabular}

NB:HFM disease=Hand-foot-mouth disease; Others=tinea pedis, allergic dermatitis, seborrheic dermatitis, atopic dermatitis, and vitiligo.

\subsection{History of Nail Biting}

Among187cases, $85(45.50 \%)$ cases had the history of the habit of biting nails.

\subsection{Location of Nonfungal Nail Disease and Serum Trace Element Result}

Location of nonfungal nail disease was assessed and the majority cases were affected on both the hands. (Figure 1). Among 187 cases sent for laboratory investigations, 76 (40.64\%) did not come with their results. However, out of the rest who underwent investigation, $30(16 \%)$ cases have trace element deficiency and the common one included zinc and iron. (Table 3)

\section{Location of nonfungal nail disease}

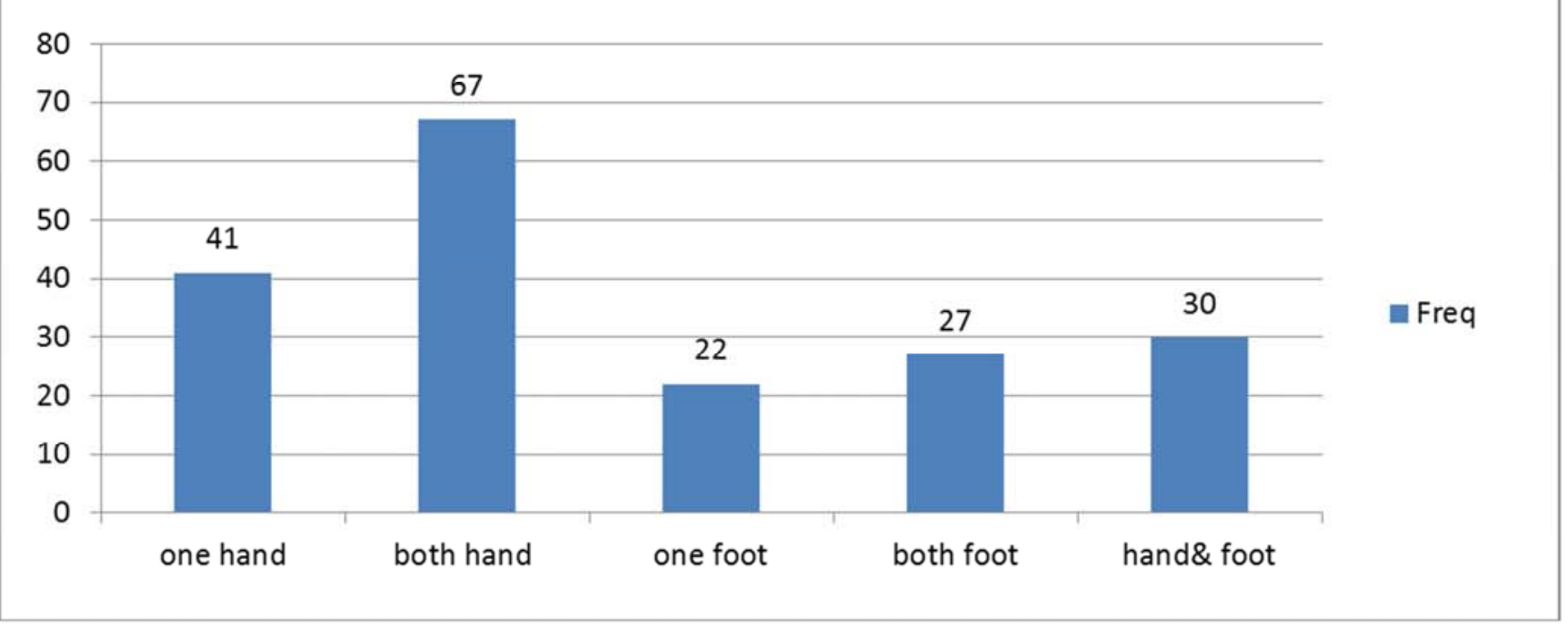

Figure 1. Location of nonfungal nail diseases among study participant. West China Hospital, Sichuan University (N=187), 2017. 
Table 3. Serum trace element examinations of study participant with nonfungal nail disease. West China Hospital, Sichuan University $(N=111)$, 2017.

\begin{tabular}{llll}
\hline Variable & Category & Frequency(N) & Percentage(\%) \\
\hline Normal limit & & 81 & 43.30 \\
& Zinc & 11 & 5.88 \\
& Iron & 6 & 3.20 \\
Element & Calcium & 7 & 3.70 \\
deficiency & Zinc and Iron & 4 & 2.13 \\
& Zinc,Calcium, and & 1 & 0.50 \\
& Iron & 1 & 0.50 \\
& Iron and Calcium & & \\
\hline
\end{tabular}

\subsection{Types of Nonfungal Nail Disease}

Among187cases, the most common type of nonfungal nail disease found in this study was onychomadesis $50(26.74 \%)$, followed by onycholysis $24(12.83 \%)$.

Table 4. Types of nonfungal nail diseases among study participant. West China Hospital, Sichuan University (N=187), 2017. (Table 4)

\begin{tabular}{lll}
\hline Variable & Frequency(N) & Percentage(\%) \\
\hline Onychomadesis & 50 & 26.74 \\
Onycholysis & 24 & 12.83 \\
Nail pitting & 31 & 16.58 \\
Nail discoloration & 9 & 4.81 \\
Trachyonychia & 27 & 14.44 \\
Lichen Planus & 7 & 3.74 \\
Nail thickening & 27 & 14.44 \\
Others & 12 & 6.42 \\
\hline
\end{tabular}

NB: Others: Beau's line, longitudinal split, longitudinal melanonychia, koilonychia, nail plate disruption, multiple transverse grooves of thumbnails, longitudinal dystrophy of the thumbnail.

\subsection{Distribution of Onychomadesis}

The distribution of 50 cases of onychomadesis was described. In majority $42(84 \%)$ was seen in age group $<5$ years. Among those cases of onychomadesis, 15 cases had the history of both nail biting and HFMD. 26 (52\%) cases had the history of the hand-foot-mouth disease. (Table 5)
Table 5. Distribution of onychomadesis among study participant. West China Hospital, Sichuan University (N=50), 2017.

\begin{tabular}{llll}
\hline Variable & Category & Frequency(N) & Percentage(\%) \\
\hline \multirow{4}{*}{ Age } & $1-5$ years & 42 & 84 \\
& 6-12 years & 7 & 14 \\
Gender & $13-18$ years & 1 & 2 \\
Habit of nail biting & Male & 27 & 54 \\
History of common & & 23 & 46 \\
cold & & 23 & 46 \\
HFMD & & 22 & 44 \\
HistoryofSkinDisease & Eemale & 26 & 52 \\
& Erticaria & 1 & 4 \\
& Others & 1 & 2 \\
& One hand & 7 & 2 \\
Location of Disease & Both hands & 27 & 14 \\
& One foot & 1 & 54 \\
& Both foot & 5 & 2 \\
& Hand foot & 10 & 10 \\
Residence & Urban & 48 & 20 \\
& Rural & 2 & 96 \\
& Kindergarten & 19 & 4 \\
Education & Primary & 9 & 38 \\
& Secondary & 1 & 18 \\
\hline & Noeducation & 21 & 2 \\
& & 42 \\
\hline
\end{tabular}

NB:HFMD=Hand-foot-mouthdisease;Others:Seborrheicdermatitis

\subsection{Factors Associated with Onychomadesis}

To identify the factors associated with onychomadesis, binary logistic regression was done on each variable to see the association and identify the variables with the $\mathrm{P}$ value less than 0.05 . Hence, variables like age, history of HFMD, skin disease showed association with onychomadesis at $(\mathrm{P}$ value 0.001), ( $\mathrm{P}$ value 0.0001$)$ and (P value 0.020) respectively. (Table 6)

Table 6. Binary logistic regression with factors associated with onychomadesis. West China Hospital, Sichuan University (N=50),2017.

\begin{tabular}{|c|c|c|c|c|c|}
\hline Variable & Category & Frequency $(N=187)$ & Frequency $(\mathrm{N}=50)$ & $\operatorname{COR}(95 \% \mathrm{CI})$ & Sig. \\
\hline Age & & 187 & 50 & $.816(.724, .920)$ & $0.001 *$ \\
\hline \multirow{2}{*}{ Gender } & Male & 91 & 27 & $1.339(.699,2.564)$ & .378 \\
\hline & Female & 96 & 23 & 1 & \\
\hline Nai lbiting & & 85 & 23 & $.928(.538,1.974)$ & .928 \\
\hline HFMD & & 44 & 26 & $7.162(3.404,15.07)$ & $.0001 *$ \\
\hline Common Cold & & 77 & 22 & $1.171(.609,2.254)$ & .636 \\
\hline \multirow{2}{*}{ Residence } & Urban & 175 & 48 & $1.890(.399,8.940)$ & .422 \\
\hline & Rural & 12 & 2 & 1 & \\
\hline \multirow{4}{*}{ Education } & Kindergarten & 52 & 19 & $1.099(.902,1.339)$ & .350 \\
\hline & Primary & 65 & 9 & 1 & \\
\hline & Secondary & 12 & 1 & 1 & \\
\hline & No education & 58 & 21 & 1 & \\
\hline
\end{tabular}

NB: HFMD= Hand-foot-mouth disease 


\subsection{Multiple Logistic Regression of Onychomadesis}

To identify the variables that have a strong association and significant variables at $\mathrm{P}$ value less than 0.05 in binary logistic regression was reanalyzed in multiple logistic regressions to identify variables which have a significant association with onychomadesis. In this test, hand-foot-mouth disease ( $\mathrm{P}$ value 0.0001$)$ was significant, children with the history of hand-foot-mouth disease were 5.8 times more likely to have onychomadesis than the counter part at $P$ value 0.0001 . And age ( $P$ value 0.006 ) was significant signifies, with the increase in age,the problem of onychomadesis is 0.833 times less likely to have. (Table 7)

Table 7. Multiple logistic regression associated with onychomadesis. West China Hospital, Sichuan University (N=50), 2017.

\begin{tabular}{llll}
\hline Variable & Frequency(N=187) & Frequency(N=50) & AOR(95\%CI) \\
\hline Age & 187 & 50 & $.833(.731, .948)$ \\
HFMD & 44 & 26 & $5.898(2.721,12.783)$ \\
Skin disease & 37 & 4 & $.006^{*}$ \\
\hline
\end{tabular}

NB:HFMD=Hand-foot-mouth disease

\section{Discussion}

Nonfungal nail disease in children can be analyzed and studied under various categories such as age, gender, education, residence, related diseases, etc to identify the magnitude of most common nail diseases prevalent in children. The study of the causes of nonfungal nail diseases in children and adolescents is complicated and challenging. Nail disorders in children is an uncommon cause of dermatological consultation, which may be congenital, hereditary or acquired [8]. Globally, there are no specific guidelines to specify the factors associated with nonfungal nail disease in children and adolescents. This study is a descriptive cross-sectional study of different types of nonfungal nail diseases and clinical analysis of nonfungal nail disease of children and adolescents done in West China Hospital of Sichuan University.

The nonfungal nail diseases include onychomadesis, onycholysis, trachyonychia, nail pitting, nail thickening, nail lichen planus, beau's line, longitudinal melanonychia, koilonychia, nail plate disruption, multiple transverse grooves of thumbnails, longitudinal dystrophy of the thumbnail.

Only a few studies about the clinical analysis of nonfungal nail diseases in children has been conducted in China and rest of the world. Similar to this study, in 2008, 23 children with nail diseases were reported and analyzed according to age, gender, and nonfungal nail diseases. [6] Recently in 2012, 170 children with nonfungal nail diseases were analyzed according to age, gender, nutrition, residence, food, location of nails, treatment of nails, habit of nail biting, associated diseases, nail diseases and serum trace element investigations [7].

In this study, the clinical characteristics of nonfungal nail diseases in children and adolescents were analyzed. The mean age of cases with nonfungal nail disease was 5.5 years $(\mathrm{SD} \pm 3.8)$. The majority of $113(64.40 \%)$ cases with nail disorders were less than five years of age, $59(31.60 \%)$ in 6-12 age group and $15(8 \%)$ in 13-18 age group. This study showed the occurrence of nonfungal nail diseases decreases with the increase in age of the children. Male 91 (48.90\%) and female $96(51.30 \%)$ were reported in this study. The male:female ratio was $0.95: 1.175(93.60 \%)$ lived in the urban area and $12(6.40 \%)$ lived in rural area. $52(27.80 \%)$ went to kindergarten, $65(34.80 \%)$ went to primary school and $12(6.4 \%)$ went to secondary school. In the previous study, it was reported that among 170 children with nonfungal nail diseases, less than 2 years were 18 and 2-6 years of age were 114 which is similar to our study where the occurrence of nonfungal nail diseases was mostly in the age group 1-5years [7]. In this study $85(45.50 \%)$ reported to have the habit of nail biting. Nail biting is most common in childhood. One study revealed that $36 \%$ of 5 years old, $57 \%$ of 12 years old and $31 \%$ of 16 years old bites nail $[9,10]$. Studies suggest that onychomadesis [11] and onycholysis [12] has been reported to be caused by traumatic injuries which may also consist nail biting.

The location of nonfungal nail diseases in children and adolescents assessed in one hand 41(21.90\%), both hands 67 (35.80\%), one foot $22(11.76 \%)$, both foot $27(4.44 \%)$ and both hand and foot 30 (16.04\%) were reported in this study. Similar to this study, in 170 children with nonfungal nail diseases, 114 cases with fingernails, 32 cases with toenails and 24 cases with both hand and foot nails were reported [7]. However, no studies with nonfungal nail diseases have been categorized into one hand, both hands, one foot, both foot and both hand and foot in the literature.

The nonfungal nail diseases were classified into onychomadesis $50(26.74 \%)$, Onycholysis $24(12.83 \%)$, nail pitting 31 (16.58\%), nail discoloration 9 (4.81\%), trachyonychia $27(14.44 \%)$, lichen planus $7(3.74 \%)$, nail thickening 27 (14.44\%) and others 12 (6.42\%) (beau's line, longitudinal split, longitudinal melanonychia, koilonychia, nail plate disruption, multiple transverse grooves of thumbnails, longitudinal dystrophy of thumbnail). In a study of 23 children with nonfungal nail disease showed 1 children with twenty nail dystrophy, 3 with onychocryptosis, 1 with onychomadesis, 3 with Beau's line, 1 with median nail dystrophy, 5 with linear nails, 4 with pitted nails and 5 with longitudinal melanonychia [6]. In another study of 170 children with nonfungal nail disease showed three most common nail disease included 31 children with nail pitting, 28 with brittle nails oronychorrhexis and 27 with leukonychia [7].

Onychomadesis is the separation of the nail from the 
proximal matrix leading to nail loss [13]. It was first described in 2000 after an outbreak in Chicago as a result of the hand-foot-mouth disease [14]. Many reports suggested the association of onychomadesis with the hand-foot-mouth disease. A recent study in China reported 56 (20.50\%) cases out of 273 cases of the hand-foot-mouth disease were identified with nail changes presented as onychomadesis [15] Onycholysis is the separation of the distal nail plate from the nail bed and due to the abnormal loss of adherence of the nail plate to the nail bed, affected nails appears white in color [16]. It is caused by finger sucking in an infant [17]. Nail pitting is defined as the presence of small depressions on the nail plate usually associated with psoriasis, alopecia areata and eczema [18]. Children with psoriatic arthritis have an association with nail pitting [19]. Nail discoloration is classified into leukonychia or white nails, longitudinal erythronychia, melanonychia, green nails, staining of the nail plate, red lunulae, spotted lunulae, purpura of the nail beds, blue nails, and yellow nail syndrome. The yellow nail syndrome is characterized by thickening and yellowish-green color of the nails associated with lymphedema and compromised respiration [20]. Pediatric Trachyonychia is an acquired disease characterized by longitudinal striations, ridges, fissures or pitting. The term 20-nail dystrophy is commonly used to describe nail dystrophy in all or most nails in children [21]. It has been associated with alopecia areata, psoriasis, lichen planus, vitiligo, and incontinentia pigmenti [22]. Nail lichen planus is very rare in children, and only 15 biopsies proved cases had been reported in the literature [23]. Tosti etal. reported that among 15 children, 10 had typical nail matrix lesions, 2 had 20-nail dystrophy (trachyonychia), and 3 had idiopathic atrophy of the nails [23]. Pterygium is the thinning and distal splitting of nail plates, associated with lichen planus. It is mainly caused by trauma, ischemia, and gangrene or severe dermatologic disease such as toxic epidermal necrolysis or lichen planus [20]. Nail thickening or onychauxis may be defined as the presence of subungual scales that lead the nail plate to be thickened.

The five most nonfungal nail diseases in children and adolescents were further analyzed and found that onychomadesis, onycholysis, nail pitting and nail thickening were mostly distributed in the age group 1-5 years and trachyonychia in the age group 6-12 and 1-5 years. It was further analyzed as there were fewer cases and no reports have been published in this regard in the literature. The characteristic features among 5 diseases on the basis of the location of nonfungal nail disease were mostly affected in both hands in onychomadesis 27 (54\%), both foots in onycholysis $6(25 \%)$ and both hand foot in onychomadesis $10(20 \%)$. The history of hand-foot-mouth disease 26 (52\%) was mostly found in onychomadesis. The habit of nail biting $23(46 \%)$ was mostly seen in onychomadesis. The history of common cold $22(44 \%)$ was mostly found in onychomadesis.

Onychomadesis $50(26.7 \%)$ was chosen to be the dependent variable in the binary logistic regression. To identify factors associated with nonfungal nail disease the binary logistic regression was performed one by one on each variable. Finally, factors like age (P value 0.001), HFMD (P value 0.001$)$ and skin disease $(P$ value 0.020$)$ were significantly associated with onychomadesis.

Finally, significant variables in binary logistic regression were entered in multiple logistic regressions to identify variables that have a significant association with onychomadesis. Then variables like age (P value 0.006$)$ and HFMD ( $\mathrm{P}$ value 0.0001) were identified to be significant. This study also suggests that the children's age in HFMD strongly shows association with onychomadesis as suggested by other literature reviews.

Wong et al. reported that hand-foot-mouth disease (HFMD) is a common childhood illness mostly seen in children younger than 10 years old [24]. In our study, 24 (57.10\%) cases age group of 1-5years had the history of hand-foot-mouth disease and onychomadesis. The association between HFMD and onychomadesis was proposed 17 years ago $[25,26]$. Finnish and Spanish authors observed a sufficient number of children developing onychomadesis approximately six weeks after they had suffered from HFMD, which makes more clear association $[27,28,29,30]$. As reported by the Chinese authors in Henan Province, China 69 children out of 73 suffering from onychomadesis had a history of HFMD [31].

This descriptive cross-sectional study not only concerns in the magnitude of nonfungal nail disease among children and adolescents age 1-18 years but also provides the distribution of nonfungal nail disease in children. Since there are no specific guidelines for distribution of nonfungal nail disease in children this cross-sectional study might provide evidence and ethical significance for future research.

\section{Conclusion}

This descriptive cross-sectional study shows the occurrence of nonfungal nail diseases decreases with the increase in the age of children and the most common nonfungal nail diseases were onychomadesis and it affected both hands. Age and hand-foot-mouth disease were found statistically significant factors associated with onychomadesis.

The limitation of this study was the descriptive cross-sectional study. The sample was taken from only one hospital and most of the cases sent for serum trace element examination did not come with their result. Finally, we recommend consecutive and strong studies will be needed in the future.

\section{Acknowledgement}

We thank Gebremeskel Mirutse for assistance with statistical analysis and for comments that significantly improved the manuscript.

\section{Abbreviations}

HFMD: Hand-foot-mouth-disease 
SPSS: Software Package for Social Science

$\mathrm{KOH}$ : Potassium hydroxide

\section{References}

[1] Chernoff K. A, Scher R. K. Nail disorders:Kids are not just little people. Clinics in Dermatology. 2016; 34:736-741.

[2] Silverman RA. Diseases of the nails in infants and children. Adv Dermatol.1990;5:153-170.

[3] Schachner L, Ling NS, Press S. A statistical analysis of a pediatric dermatology clinic. Pediatr Dermatol. 1983; 1:157-164.

[4] Iglesias A, Tamayo L, Sosa-de-Martinez C, etal. Prevalence and nature of nail alterations in pediatric patients. Pediatr Dermatol. 2001; 18:107-109.

[5] Fan WG. Clinical study of 98 cases of nail disease. China J Dermatol. 2006; 39(10):607-607. (In Chinese).

[6] Zou XY. Clinical analysis of 23 children with nonfungal nail disease. China J Le Dr Skin Dis. 2008; 24(10):842-842. (In Chinese).

[7] Yin RR, Wang XR ,Xian XS, Zhang CL,Wei SH. Clinical analysis of 170 children with nonfungal dystrophy of nail. J Clin Dermatol. 2012; 41(12):721-722(InChinese).

[8] Piraccini BM and Starace M. Nail disorders in infants and children. Curr Op in Pediatr. 2014; 26:440-445.

[9] Baran R, Dawber RPR, deBerker DARetal. Barun and Dawber's diseases of the nails and their management. $3^{\text {rd }}$ ed. Oxford: Blackwell Science, 2001.

[10] deBerker DAR, Baran R, Dawber RPR. Handbook of diseases of the nails and their management. Oxford: Blackwell Science, 1995.

[11] Peterson RR, Spurgeon R, Lakshmi KS, Rath S. Onychomadesis in a healthy child. Indian J Paediatr Dermatol. 2015; 16:264-5.

[12] Zaias N, Escovar S. X, Zaiac M. N. Finger and toenail Onycholysis. European Academy of Dermatology and Venereology. 2015; 29:848-853.

[13] Laure J, MD ,Sarah Z, MD and Jacob L, MD. Mao. Nail findings in hand-foot-and-mouth disease. The Pediatric Infectious Disease Journal. 2015; 34(4):449-450.

[14] Clementz GC, Mancini AJ. Nail matrix arrest following hand-foot-mouth disease: a report of five children. Pediatr Dermatol. 2000; 17:7-8.

[15] Long DL, Zhu SY, Li CZ, Chen CY, Du WT, Wang X. Late-onset nail changes associated with hand,foot,and mouth disease:A clinical analysis of 56 cases. Pediatr Dermatol. 2016; 33(4):424-428.

[16] ShahKara N and Rubin Adam I. Nail Disorders as Signs of
Pediatric systemic disease. Curr Probl Pediatr Adolesc Health Care. 2012; 42:201-211.

[17] Wu E, Viegas SF. Finger sucking and Onycholysis in an infant J Hand Surg Am. 2005; 30:620-2.

[18] Tosti A and Piraccini BM. Nail Disorders. Hair, Nails and Mucous Membranes. Dermatology. $3^{\text {rd }}$ Ed. Elsevier Saunders. 2012:1129-1147.

[19] Barth JH, Dawber RP. Diseases of the nails in children. Pediatr Dermatol. 1987; 4:275-290.

[20] James W D, Berger TG, Elston DM. Disease of the nails. Diseases of the Skin appendages. Andrew's Diseases of the skin: clinical dermatology. 11ed. USA. Saunders Elsevier. 2011:741-782.

[21] Kumar MG, Ciliber to Hand Bay liss SJ. Long-term follow up of pediatric Trachyonychia. 2015; 32:198-200.

[22] Sehgal VN. Twenty nail dystrophy trachyonychia: an overview. J Dermatol. 2007; 34:361-366.

[23] Tosti A, Piraccini BM, Cambiaghi S, Jorizzo M. Nail lichen planus in children clinical features, response to treatment and long term follow-up. Arch Dermatol. 2001; 137:1027-1032.

[24] Wong SS, Yip CC, Lau SK, etal. Human enterovirus 71 and hand, foot and mouth disease. Epidemiol Infect. 2010; 138:1071-1089.

[25] Clementz GC, Mancini AJ. Nail matrix arrest following hand-foot-mouthdisease report: a report of five children. Pediatr Dermatol. 2000; 17(1):7-11.

[26] BernierV, Labreze C, Bury F, Taleb A. Nail matrix arrest in the course of hand, foot and mouth disease. Eur J Pediatr. 2001; 160(11):649-51.

[27] Osterback R, Vuorinen T, Linna M, Susi P, Hyypia T, Waris M. Coxsackievirus A6 and hand, foot, and mouth disease, Finland Emerg Infect Dis. 2009; 15(9):1485-8.

[28] Redondo Grando MJ, Torres Hinojal MC, Izquierdo Lopex B. Brotedeonicoma desisposviricaen Valladolid. [Postviral Onychomadesisout breakin Valladolid]. Spanish. AnPediatr (Barc). 2009; 71(5):439-9.

[29] Blomqvist S, Klemola P, Kaijalainen S, Paananen A, Simonen ML,Vuorinen T, etal. Co-circulation of coxsackieviruses A6 and Also in hand, foot and mouth disease outbreak in Finland. J Clin Virol. 2010; 48(1):49-54.

[30] Davia JL, Bel PH, Ninet VZ, Bracho MA, Gonzalez-Candelas F, Salazar A, Gobernado M, Bosch IF. Onychomadesis outbreak in Valencia, Spain associated with hand, foot and mouth disease caused by enterviruses. Pediatr Dermatol. 2011; 28(1):1-5.

[31] TANG Jian-Ping, HU Meng-Ye, WEI Zhu. Clinical features of onychomadesis following hand-foot-mouth disease in children. China J Contemp Pediatr. 2014; 16(12):1275-1276. [In Chinese]. 